Article

\title{
A city-scale optimization for distribution of goods with stochastic service
}

\author{
Manuel Vargas ${ }^{1}$, Sebastián Dávila², Miguel Alfaro1 ${ }^{1}$, Guillermo Fuertes ${ }^{1,3}$ and Marc Dahmen ${ }^{1, *}$ \\ 1 Departamento de Ingeniería Industrial, Universidad de Santiago de Chile, Santiago, Chile \\ 2 Departamento Ingeniería Industrial, Universidad de Chile, Chile \\ 3 Facultad de Ingeniería, Ciencia y Tecnología, Universidad Bernardo O’Higgins, Chile
}

\begin{abstract}
The research develops the vehicular routing problem for the distribution of refrigerated products in a multinational company. The mathematical model corresponds to the vehicle routing problem with hard time windows and stochastic service (VRPTW-ST) time model applied in the city of Santiago de Chile. For optimization of the model, optimization methods tabu search, chaotic search and general algebraic modeling were used. The results allow to validate the meta-heuristic chaotic search to solve the VRPTW-ST; chaotic search method obtains superior performance than tabu search method for solving a real problem in a large city.
\end{abstract}

Keywords: vehicle routing problem; Metaheuristics; VRPTW-ST

\section{Introduction}

Between the 10 and the $20 \%$ of the cost of sale of a product is associated to the logistic chain, specifically to the transport cost of the products of which the $20 \%$ is related with the vehicle routing problem (VRP) [1]. According to Toth and Vigo [2] the reduction in distribution times generates an increase in sales and improves the participation of industrial organizations in the markets; at the same time the decrease in costs is reflected in the increase in profits [3].

VRPTW-ST sets new restrictions on the VRP model; time windows limits (TW) are constraints and the service times (ST) are random variables [4,5]. Other additional features of the general VRPTW-ST model are (1) the vehicles can arrive early at the opening time, however, the reception will take place within the time window with random reception time. The complexity of the VRPTW-ST model lies in the probability of distribution of the arrival of the vehicles generated by the TW restrictions and the ST variables [6].

The investigation addresses a VRPTW-ST in a frozen products distribution center of a transnational food company located in the Quilicura commune in the Metropolitan Region of Santiago. Daily the distribution routes are planned to meet the demand of customers located in the same region. For this case study, two customer segments are considered: (1) supermarkets and (2) road customers. The former establishes a deadline for receiving orders, that is, the truck is not serviced when it arrives after the scheduled time. Another restriction of the supermarkets is the random waiting time of the trucks before being served. In the case of road customers, they can receive the order at any time and once the trucks arrive they immediately begin the process of receiving the merchandise [7].

Finally, hybrid methods allow solving VRPTW-ST. The two-phase method solves a model without the possibility of failures to obtain a preliminary result and subsequently resolves a second model that considers the costs associated with the failure of any of the restrictions. Authors such as [8] use a portfolio of evolutionary algorithms to shorten computing time in the search for local solutions. 


\section{Problem Statement}

The problem described above can be represented as a VRP with deadline and stochastic waiting time (VRPTW-ST) for a real problem of distribution of frozen food of a transnational company in the City of Santiago. The vehicle is serviced in the intervals of attention time defined by the client and the waiting time is defined by a stochastic time function. Based on previous works [9], the research proposed a mathematical programming formulation with probabilistic restrictions. This model considers the random nature of the problem when establishing a level of service for each customer [10]. To address the complexity of the VRPTW-ST, [9] develops a model based on meta-heuristic TS and the authors [11] use a chaotic search (CS) method obtaining similar or better results than using the TS method.

For the validation of the proposed methodology, models were constructed to compare both methods with the empirical results of the Industry. The novelty of this research is the development of a metaheuristic that solves real VRPTW-ST for the distribution of merchandise in large cities. Currently these problems cannot be solved in adequate times by the general algebraic modeling (GAMS) technique. In this investigation to solve the problem of time and computational capacity, the use of CS and TC heuristics is proposed.

\section{Simulations}

The VRPTW-ST model probability restrictions are based on the author's work [12] and allow assigning service levels to comply with the probability restrictions. Model variables:

$$
\begin{aligned}
& x_{i j k}\left\{1 \text { if the vehicle } k \text { travels from customer } i \quad k \in K, \quad i, j \in V_{o}\right. \\
& \text { to customer } j, 0 \text { in other case })
\end{aligned}
$$

\subsection{Objective Function}

$$
\min _{0}(x)=M \sum_{j \in V_{0}} \sum_{k \in K} x_{0 j k}+\sum_{i \in V} \sum_{j \in V} \sum_{k \in K} d_{i j} x_{i j k}
$$

\subsection{Constraints}

$$
\begin{array}{cc}
s . a \cdot \sum_{k \in K} \sum_{j \in V} x_{i j k}=1 & \forall i \in V \backslash(0, n+1\} \\
\sum_{j \in V} x_{0 j k}=1 & \forall k \in K \\
\sum_{i \in V} x_{i n+1 k}=1 & \forall k \in K \\
\sum_{i \in V_{0}} x_{i j k}-\sum_{i \in V_{0}} x_{j i k}=0 & k \in K, j \in V_{0} \\
\sum_{i \in V_{0}} q_{i} \sum_{j \in V} x_{i j k} \leq Q & \\
s_{j k}-s_{i k} \geq t_{i j k}+\delta_{i}+w_{i}-M\left(1-x_{i j k}\right) & \\
& \\
&
\end{array}
$$




$$
\begin{gathered}
P\left(\sum_{i \in V} \sum_{j \in V} t_{i j} x_{i j k}+\sum_{i \in V_{0}}\left(\delta_{i}+w_{i}\right) \sum_{j \in V} x_{i j k} \leq B\right) \geq \beta \quad \forall k \in K \\
P\left(\sum_{i \in V_{0}} \sum_{j \in V_{0}} w_{i} x_{i j k} \leq W\right) \geq \gamma \quad \forall k \in K \\
x_{i j k} \in(0,1\} \quad \forall(i, j) \in V, k \in K \\
s_{i k} \geq 0 \quad \forall i \in V, k \in K
\end{gathered}
$$

Equation (1) represents the objective function, where parameter $\mathrm{M}$ is a large value to ensure the minimization of the number of vehicles and the total distance traveled. Equation (2) represents that each customer can only be visited once by a vehicle. Equations (3) and (4) indicate that the vehicle $\mathrm{k}$ must leave and return to the depot. Restriction (5) forces each vehicle $\mathrm{k}$ to leave the node. Equation (6) represents the capacity constraint. Equations (7) and (8) are the stochastic restrictions of driving time and waiting time of vehicles using the decision variable $x_{i j k}$. Equation (9) represents the possibility of arriving from the vehicle before the deadline $\left(l_{n+1}\right)$ with a level of confidence $\alpha$. Equation (10) represents the maximum waiting time of each vehicle. The model prioritized the customer to reduce the difficulty of finding a solution. Finally, the model is composed of the following sets, parameters and variables (table 1$)$.

\subsection{Improvement stochastic restrictions.}

Equation (10) is a new restriction to the original model proposed by [9] and restricts the extra times of each vehicle by adding up all the waiting times of each vehicle $W$ under a $\gamma$ probability.

Equation (8) is transformed into several restrictions where a certain number of possible scenarios are evaluated. The purpose of the transformation is to meet these restrictions a minimum number of times and the compliance number will depend on the level of trust present in the original restriction.

For this, the set of scenarios is defined as $s=\left(s_{1}, \ldots, s_{s c e n}\right)$ where $s_{\text {scen }}$ is the number of scenarios. The $\mathrm{w}_{i}$ variable must be redefined by $\mathrm{w}_{i s}$ to represent the customer's wait time $i$ on stage. The variable $y_{s}$ is a binary variable with value 1 when the constraint (8) is satisfied and 0 otherwise. Meanwhile, $\pi^{\mathcal{S}}$ is the probability of occurrence of the scenarios; in this case it is assumed that all the scenarios have the same probability. Finally, $c c_{1}$ is the probability of failure of the constraint (8) must be greater than zero and less than the probability P. Equation (8) can be written as the following set of restrictions.

$$
\begin{gathered}
\sum_{i \in V_{0}} \sum_{j \in V_{0}} w_{i s} x_{i j k} \leq W+M\left(1-y_{s}\right) \quad \forall k \in K, \forall s \in S \\
\sum_{i \in V_{0}} \sum_{j \in V_{0}} w_{i s} x_{i j k} \leq W-M y_{s} \quad \forall k \in K, \forall s \in S \\
c c_{1}=1-\sum_{s \in S} \pi^{s} y_{s} \quad \pi^{s}=\frac{1}{\operatorname{card}(S)} \\
0 \leq c c_{1} \leq(1-\gamma)
\end{gathered}
$$


Table 1: Set, parameters and variables of model

\begin{tabular}{ll}
\hline Variable & Set and parmeters \\
\hline$m$ & $:$ number of vehicles used, can be constant or a decision variable. \\
$\mathrm{nk}$ & $:$ number of customers served by the vehicle $\mathrm{k}$. \\
$\mathrm{K}$ & $:$ set of vehicles, which is defined as $=(1,2, \ldots, m\}$. \\
$\mathrm{Q}$ & $:$ vehicle capacity; assumed that all the vehicles are homogeneous and each one has limited capacity \\
$\mathrm{Q}=3000 \mathrm{Kgm}$ & $:$ established working time of the driver. In this case it is added with a value of 480 min. The value of \\
$\mathrm{B}$ & B is composed of travel time, service time and waiting time. \\
& $:$ customer demand $\mathrm{i} \in \mathrm{V}_{o}$. \\
$q_{i}$ & $:$ distance between vertex $\mathrm{i}$ and $\mathrm{j}$, with $\mathrm{i}, \mathrm{j} \in \mathrm{V}$. \\
$d_{i j}$ & $:$ travel time between vertex $\mathrm{i}$ and $\mathrm{j}$. \\
$t_{i j}$ & $:$ customer service time $\mathrm{i}$. \\
$\delta_{i}$ & $:$ customer timeout $\mathrm{i} ;$ in this work, $w_{i}$ is assumed as a continuous random variable with normal \\
$w_{i}$ & distribution $w_{i} \sim N\left(\mu_{i,} \sigma_{i}^{2}\right)$. \\
$\mathrm{A}$ & $:$ maximum waiting time for vehicle $\mathrm{k}$. \\
\hline
\end{tabular}

$$
y_{s} \in(0 k, 1) \quad \forall s \in S
$$

Finally, the model plans a set of routes subject to probability restrictions, such scenarios allow routes not satisfied under a certain threshold. However, it does not consider the costs associated with the failure of routes. In this case, the objective is to minimize the number of vehicles and the total distance traveled; for Sawik [13] solutions with fewer vehicles are better than solutions with less total distance traveled.

\section{Results}

The investigation corresponds to the resolution of a real VRPTW-ST product transport problem in the City of Santiago. To validate the implementation of the TS and CS methods, they were compared with the authors' works [12]. The experiment was broaden with instances between 10 to 80 customers for the percentages of stochastic customers 10\%, 20\% and $30 \%$. The resolution of the problem was made with TS and CS. For the TS and CS methods, the results of the comparison of the total travel distance are presented (Table 1). The results establish an improvement of the CS with respect to TS with the increase in the number of nodes. The CS method has a greater capacity for diversification allowing avoiding local minimums.

\subsection{Case Ice-Cream Industry Results.}

The city of Santiago, Chile is in a valley of $15.000 \mathrm{~km}^{2}$. The big customers of the Ice-Cream Industry are 157 establishments with 43 customers with hard time windows and stochastic service. The large number of customers generates a low quality solution using the GAMS method. The GAMS programming was carried out by creating subgroups throughout the different areas of Santiago, the total post-resolution time exceeds 20 hours, a daily excess of 22-hour deadline is incurred with the truck fleet and it is possible not to exceed the normal working hours of the drivers.

The results obtained with the TS and CS algorithms for the Ice-Cream Industry problem show a (1) clear decrease in processing time; (2) reduce the distance traveled; (3) do not exceed the deadline, and (4) do not they generate extra work. The results delivered by TS and CS increase the overall quality of service by reducing the deadlines.

\section{Conclusions}

In this work, the VRPTW-ST model is used to solve a routing problem in the city of Santiago de Chile, specifically in the distribution center of the Ice-cream area of the Unilever Company. The utilization of the TS and CS optimization methods were validated 
Table 2: Comparison of total traveled distance between both methods, supermarket \%.

\begin{tabular}{|c|c|c|c|c|c|c|c|c|c|}
\hline & $10 \%$ & & & $20 \%$ & & & $30 \%$ & & \\
\hline Customer & Chaos & Tabu & Difference & Chaos & Tabu & Difference & Chaos & Tabu & Difference \\
\hline 10 & 82.65 & 82.42 & $0 \%$ & 88.37 & 85.82 & $3 \%$ & 132.64 & 131.36 & $1 \%$ \\
\hline 20 & 136.30 & 156.89 & $-13 \%$ & 132.00 & 143.66 & $-8 \%$ & 175.53 & 183.95 & $-5 \%$ \\
\hline 30 & 158.90 & 145.78 & $-9 \%$ & 236.44 & 215.6 & $10 \%$ & 225.43 & 230.33 & $-2 \%$ \\
\hline 40 & 196.20 & 199.55 & $-2 \%$ & 220.45 & 230.33 & $-4 \%$ & 326.45 & 337.42 & $-3 \%$ \\
\hline 50 & 282.29 & 318.5 & $-11 \%$ & 402.24 & 414.81 & $-3 \%$ & 391.32 & 408.60 & $-4 \%$ \\
\hline 60 & 326.61 & 355.71 & $-8 \%$ & 394.22 & 411.42 & $-4 \%$ & 494.76 & 504.51 & $-2 \%$ \\
\hline 70 & 369.75 & 408.35 & $-9 \%$ & 446.68 & 477.53 & $-6 \%$ & 496.11 & 522.23 & $-7 \%$ \\
\hline 80 & 398.20 & 439.22 & $-9 \%$ & 495.21 & 510.32 & $-3 \%$ & 581.32 & 593.69 & $-2 \%$ \\
\hline
\end{tabular}

by comparison with the GAMS algorithm. The results allowed validating the use of CS for VRPTW-ST and the resolution capacity of the CS algorithm for problems with stochastic time-out variable is established. The CS algorithm obtained better results compared to TS.

A model with probability restrictions with respect to a model with deterministic variables (1) requires more time for resolution; (2) reduces the maximum number of customers in the model, and (3) increases the distances and vehicles required for resolution. The uncertainty of the waiting times generates an increase in the calculation times of the optimal solution; if the number of customers increases with stochastic waiting times, the complexity of the model increases. This model uncertainty must be controlled using different levels of services in $\mathrm{F}(\mathrm{x})$ to categorize each customer.

To validate the implementation of the TS and CS methods, the results were compared with the works of the authors $[11,14]$ were compared. The adjustment of the TS and CS parameters allowed improving convergence and achieving better results compared to the GAMS algorithm. The improvement in the performance in the distribution of refrigerated elements of the Unilever Company is achieved by the best search features of the CS method for VRPTW-ST models, (1) compliance with restrictions without decreasing service levels; (2) the ability to plan routes in shorter times; (3) shorter total routes, and (4) reducing cost overruns by controlling overtime.

The experiments were performed comparing real models with a size limit of 20 customers for the three optimization techniques. The optimization model developed in the research, using the GAMS technique with sizes greater than 20 customers cannot be solved in adequate times for operational decision-making. The applicability limits of the experiment were restricted to 80 customers using the CS optimization techniques. The growth of processing times and computer memory generates operating restrictions for the model with client size equal to or less than 100.

Finally, it should be noted that the use of efficient mathematical models positively influences the correct daily decision making of a company. This type of optimization tools facilitates the work of administrators, reducing costs and above all time; allowing to adjust changes quickly before possible eventualities that huddle in the logistic chain.

\section{Disclosure statement}

The authors declare that there is no conflict of interests regarding the publication of this paper.

Acknowledgments: Manuel Vargas thanks FONDECYT (Chile) grant No.11200993 for financial support.

\section{References}

1. Farahani, R.; Rezapour, S.; Kardar, L. Logistics operations and management : concepts and models; Elsevier: United States, 2011.

2. Toth, P.; Vigo, D., 2014.

3. Hervert-Escobar, L.; López-Ramos, F.; Esquivel-Flores, O.A. Integrated Approach to Assignment, Scheduling and Routing Problems in a Sales Territory Business Plan. Procedia Computer Science 2016, 80, 1887-1896.

4. Elshaer, R.; Awad, H. A taxonomic review of metaheuristic algorithms for solving the vehicle routing problem and its variants. Computers $\mathcal{E}$ Industrial Engineering 2020, 140, 106242-106242. 
5. Oyola, J.; Arntzen, H.; Woodruff, D.L. The stochastic vehicle routing problem, a literature review, part I: models. EURO Journal on Transportation and Logistics 2018, 7, 193-221.

6. Errico, F.; Desaulniers, G.; Gendreau, M.; Rei, W.; Rousseau, L.M. The vehicle routing problem with hard time windows and stochastic service times. EURO Journal on Transportation and Logistics 2018, 7, 223-251.

7. Pureza, V.; Morabito, R.; Reimann, M. Vehicle routing with multiple deliverymen: Modeling and heuristic approaches for the VRPTW. European Journal of Operational Research 2012, 218, 636-647.

8. Shukla, N.; Choudhary, A.K.; Prakash, P.K.S.; Fernandes, K.J.; Tiwari, M.K. Algorithm portfolios for logistics optimization considering stochastic demands and mobility allowance. International Journal of Production Economics 2013, 141, 146-166.

9. Li, X.; Tian, P.; Leung, S.C.H. Vehicle routing problem with stochastic travel times including soft time windows and service costs. International Journal of Production Economics 2010, 125, 137-145.

10. Ehmke, J.F.; Campbell, A.M.; Urban, T.L. Ensuring service levels in routing problems with time windows and stochastic travel times. European Journal of Operational Research 2015, 240, 539-550.

11. Ikeguchi, T.; Hasegawa, M.; Kimura, T.; Matsuura, T.; Aihara, K. Theory and applications of chaotic optimization methods. Innovative Computing Methods and Their Applications to Engineering Problems 2011, pp. 131-161.

12. Berhan, E.; Beshah, B.; Kitaw, D.; Abraham, A. Stochastic Vehicle Routing Problem: A Literature Survey. Journal of Information E Knowledge Management 2014, 13, 1450022-1450022.

13. Sawik, B. Weighted-sum approach for bi-objective optimization of fleet size with environmental aspects. Applications of Management Science 2018, pp. 101-116.

14. Binart, S.; Dejax, P.; Gendreau, M.; Semet, F. A 2-stage method for a field service routing problem with stochastic travel and service times. Computers $\mathcal{E}$ Operations Research 2016, 65, 64-75. 